\title{
Near-optimum Universal Graphs for Graphs with Bounded Degrees
}

\author{
(Extended Abstract)
}

\author{
Noga Alon ${ }^{1, \star}$, Michael Capalbo ${ }^{2, \star \star}$, Yoshiharu Kohayakawa ${ }^{3, \star \star \star}$, \\ Vojtěch Rödl ${ }^{4, \dagger}$, Andrzej Ruciński ${ }^{5, \ddagger}$, and Endre Szemerédi ${ }^{6, \S}$ \\ 1 Department of Mathematics, Raymond and Beverly Sackler Faculty of Exact \\ Sciences, Tel Aviv University, Tel Aviv, Israel \\ 2 Department of Mathematical Sciences, The Johns Hopkins University, \\ 3400 N. Charles Street, Baltimore, MD \\ ${ }^{3}$ Instituto de Matemática e Estatística, Universidade de São Paulo, Brazil \\ 4 Department of Mathematics and Computer Science, Emory University, Atlanta \\ 5 Faculty of Mathematics and Computer Science, Adam Mickiewicz University, \\ Poznań, Poland \\ ${ }^{6}$ Department of Computer Science, Rutgers University, NJ
}

\begin{abstract}
Let $\mathcal{H}$ be a family of graphs. We say that $G$ is $\mathcal{H}$-universal if, for each $H \in \mathcal{H}$, the graph $G$ contains a subgraph isomorphic to $H$. Let $\mathcal{H}(k, n)$ denote the family of graphs on $n$ vertices with maximum degree at most $k$. For each fixed $k$ and each $n$ sufficiently large, we explicitly construct an $\mathcal{H}(k, n)$-universal graph $\Gamma(k, n)$ with $O\left(n^{2-2 / k}(\log n)^{1+8 / k}\right)$ edges. This is optimal up to a small polylogarithmic factor, as $\Omega\left(n^{2-2 / k}\right)$ is a lower bound for the number of edges in any such graph.

En route, we use the probabilistic method in a rather unusual way. After presenting a deterministic construction of the graph $\Gamma(k, n)$, we prove, using a probabilistic argument, that $\Gamma(k, n)$ is $\mathcal{H}(k, n)$-universal. So we use the probabilistic method to prove that an explicit construction satisfies certain properties, rather than showing the existence of a construction that satisfies these properties.
\end{abstract}

\section{Introduction and Main Result}

For a family $\mathcal{H}$ of graphs, a graph $G$ is $\mathcal{H}$-universal if, for each $H \in \mathcal{H}$, the graph $G$ contains a subgraph isomorphic to $H$. Thus, for example, the complete

* Partially supported by a USA-Israeli BSF grant, by the Israel Science Foundation and by the Hermann Minkowski Minerva Center for Geometry at Tel Aviv University.

** Supported by NSF grant CCR98210-58 and ARO grant DAAH04-96-1-0013.

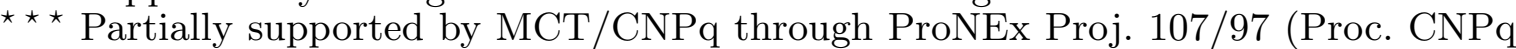
664107/1997-4), by CNPq (Proc. 300334/93-1, 468516/2000-0, and 910064/997), and by FAPESP (Proj. 96/04505-2).

$\dagger$ Partially supported by NSF grants DMS 0071261 and INT 0072064.

$\ddagger$ Supported by KBN grant 2 P03A 03216 . Part of this research was done during the fifth author's visit to Emory University.

$\S$ Partially supported by the NSF. 
graph $K_{n}$ is $\mathcal{H}_{n}$-universal, where $\mathcal{H}_{n}$ is the family of all graphs on at most $n$ vertices. The construction of sparse universal graphs for various families arises in the study of VLSI circuit design, and has received a considerable amount of attention.

For example, as discussed in [5], page 308, universal graphs are of interest to chip manufacturers. It is very expensive to design computer chips, but relatively inexpensive to make many copies of a computer chip with the same design. This encourages manufacturers to make their chip designs configurable, in the sense that the entire chip is prefabricated except for the last layer, and a final layer of metal is then added corresponding to the circuitry of a customer's particular specification. Hence, most of the design costs can be spread out over many customers. We may view the circuitry of a computer chip as a graph, and may also model the problem of designing chips with fewer wires that are configurable for a particular family of applications as designing smaller universal graphs for a particular family of graphs.

Also, as discussed in [12], we may model data structures and circuits as graphs. The problem of designing, say, an efficient single circuit that can be specialized for a variety of other circuits can be viewed as constructing a small universal graph. With these applications in mind, we note that, given a family $\mathcal{H}$ of graphs, it is often desirable to find an $\mathcal{H}$-universal graph with small number of edges.

Motivated by such practical applications, universal graphs for several different families of graphs have been studied by numerous researchers since the 1960s. For example, extensive research exists on universal graphs for forests [4], [7], [8], [9], [10], [13], and for planar and other sparse graphs [1], [3], [4], [6], [11], $[16]$.

Here we construct near-optimum universal graphs for families of boundeddegree graphs. More specifically, for all positive integers $k$ and $n$, let $\mathcal{H}(k, n)$ denote the family of all graphs on $n$ vertices with maximum degree at most $k$. By the size of a graph we always mean the number of its edges. Several techniques were introduced in [2] to obtain, for fixed $k$, both randomized and explicit constructions of $\mathcal{H}(k, n)$-universal graphs of size $O\left(n^{2-\frac{1}{k}} \log ^{1 / k} n\right)$, thereby setting a new upper bound for the minimum possible size of an $\mathcal{H}(k, n)$-universal graph. In addition, a (simple) lower-bound of $\Omega\left(n^{2-\frac{2}{k}}\right)$ was also established. However, closing the gap between the upper and lower bounds was left as an open problem.

Here we almost completely close this gap by presenting an explicit construction of an $\mathcal{H}(k, n)$-universal graph $\Gamma(k, n)$ of size $O\left(n^{2-\frac{2}{k}} \log ^{1+8 / k} n\right)$. We describe the construction of $\Gamma(k, n)$ in the next paragraph.

Construction of $\Gamma(k, n)$ : Let us set $q \equiv \log \left(k n / 8 \log ^{4} n\right)$, and $s=q / k$; so $q=$ $\log n-4 \log \log n+O(1)$, and $s$ is just slightly smaller than $(\log n) / k$. For the sake of simplicity let us omit all floor and ceiling signs, and assume that $s$ and $q$ are integers; this will not affect our arguments. Unless otherwise stated, our logarithms are to the base 2. Let $\Gamma^{\prime}(k, n)=\Gamma^{\prime}$ be the graph with the vectors in $\{0,1\}^{q}$ as its vertex-set; two vertices $v$ and $w$ are adjacent in $\Gamma^{\prime}$ if and only 
if there exist two distinct indices $j^{\prime}, j^{\prime \prime} \in\{1,2, \ldots, k\}$ such that the $(t k+j)$-th coordinate of $v$ agrees with the $(t k+j)$-th coordinate of $w$, for all but at most one of the pairs of integers $(j, t)$, where $j=j^{\prime}, j^{\prime \prime}$ and $t \in\{0, \ldots, s-1\}$. To form $\Gamma(k, n)$ from $\Gamma^{\prime}(k, n)$, replace each vertex $v$ in $\Gamma^{\prime}$ with a clique $V_{v}$ of $64 q^{4} / k=$ $\Theta\left((\log n)^{4} / k\right)$ vertices, and interconnect each vertex of $V_{v}$ with each vertex of $V_{w}$ if and only if the pair $v w$ is an edge of $\Gamma^{\prime}$.

Note that, for each fixed $k \geq 3$, the graph $\Gamma(k, n)$ has size at most

$$
\frac{k n}{16 \log ^{4} n}\left(\begin{array}{l}
k \\
2
\end{array}\right)(2 s) 2^{q-2 s+2}\left(\frac{64 q^{4}}{k}\right)^{2}=O\left(n^{2-\frac{2}{k}}(\log n)^{1+8 / k}\right),
$$

and only about $8 n$ vertices. Our main result is the following theorem.

Theorem 1. The graph $\Gamma(k, n)$ is $\mathcal{H}(k, n)$-universal for all $k \geq 3$, and $n$ sufficiently large.

The rest of this extended abstract is organized as follows. In $\S 2$, we apply a graph embedding technique to prove that $\Gamma(k, n)$ is $\mathcal{H}(k, n)$-universal, provided each member of $\mathcal{H}$ satisfies a certain decomposition property (see Lemma 1 , below). This decomposition property is easily satisfied by all graphs in $\mathcal{H}(k, n)$ for $k$ even, and by all graphs in $\mathcal{H}(k, n)$ for $k$ odd with chromatic index $k$, including all bipartite members of $\mathcal{H}(k, n)$ (see Examples 2.3 and 2.4).

The remaining case, i.e. $k$ odd and $H$ of chromatic index $k+1$ is, however, quite troublesome. In $\S 3$ we sketch a proof of the existence of a suitable decomposition of every graph $H \in \mathcal{H}(k, n)$. Finally, in $\S 4$ we show how to turn $\Gamma(k, n)$ into an $\mathcal{H}(k, n)$-universal graph $\Lambda(k, n)$ that has, say, only $(1+\epsilon) n$ vertices, and still only $O\left(n^{2-\frac{2}{k}}(\log n)^{1+8 / k}\right)$ edges.

The techniques in $\S 2$ combine combinatorial and probabilistic ideas, the proofs in $\S 3$ are based on tools from matching theory, including Tutte's Theorem and the Gallai-Edmonds Structure Theorem, while the result in $\S 4$ is obtained by applying some of the known constructions of expanders and concentrators. In order to make this abstract more complete, we present some of the more technical parts of $\S 3$ in an appendix.

\section{A Graph Embedding Technique}

A graph $F$ is $(m, M)$-path-separable if there exists a collection $\mathcal{P}$ of edge disjoint paths in $F$, each of length between $2 m$ and $4 m$ such that for every $E \subseteq E(F)$ which intersects each $P \in \mathcal{P}$, every connected component of $F \backslash E$ has fewer than $M$ vertices. Thus a graph is path-separable if we may pick in it a collection of short edge-disjoint paths with the property that any transversal of the edge sets of these paths breaks up the graph into components of bounded size.

Example 2.1. Every union $F$ of vertex disjoint paths and cycles is $(m, 8 m)$ path-separable for all positive integers $m$. Indeed, partition the edge set of each component of $F$ containing at least $4 \mathrm{~m}$ edges into paths of lengths between $2 \mathrm{~m}$ and $4 m$. 
Example 2.2. A graph in $\mathcal{H}(k, n)$ obtained from a union of vertex disjoint paths and cycles (called later units) by designating one of its components as the central unit and connecting some of the other components to the center, each by exactly one edge (called a spoke), will be called a windmill. It is easy to see that every windmill is $\left(\mathrm{m}, 64 \mathrm{~km}^{2}\right)$-path-separable. Again, partition every unit containing at least $4 m$ edges into paths of lengths between $2 m$ and $4 m$. After cutting the paths with a set $E$, each vertex of the largest piece (of order at most $8 m$ ) in what is left of the central unit can be connected with up to $k-1$ paths from the other units, each of length less than $8 \mathrm{~m}$.

A graph $H \in \mathcal{H}(k, n)$ is $(2, k, m, M)$-decomposable if one can find subgraphs $F_{1}, \ldots, F_{k}$ of $H$, not necessarily all distinct, such that each $F_{i}$ is $(m, M)$-pathseparable, and each edge of $H$ appears in exactly 2 subgraphs $F_{i}$. Let us call $F_{1}, \ldots, F_{k}$ a $(2, k, m, M)$-decomposition of $H$.

Example 2.3. Every graph $H \in \mathcal{H}(k, n)$ of chromatic index $k$ is $(2, k, m, 8 m)$ decomposable into graphs from $\mathcal{H}(2, n)$. Indeed, let $M_{1}, \ldots, M_{k}$ be matchings that cover the edges of $H$. Let $F_{1}=M_{1} \cup M_{2}, F_{2}=M_{2} \cup M_{3}, \ldots, F_{i}=M_{i} \cup$ $M_{i+1}, \ldots, F_{k-1}=M_{k-1} \cup M_{k}, F_{k}=M_{k} \cup M_{1}$.

Example 2.4. If $k$ is an even integer, then every graph $H \in \mathcal{H}(k, n)$ is $(2, k, m$, $8 m$ )-decomposable into graphs from $\mathcal{H}(2, n)$. This time, by the Petersen Theorem (see, e.g., [15], p. 218), every such graph can be covered by $k / 2$ subgraphs $F_{1}, \ldots, F_{k / 2}$, where $F_{i} \in \mathcal{H}(2, n)$ for all $i$. Set $F_{k / 2+j}=F_{j}, j=1, \ldots, k / 2$.

As far as we know, for odd $k$, it is still open as to whether or not every graph $H \in \mathcal{H}(k, n)$ has a $(2, k, m, O(m))$-decomposition. However, for all integers $k$ and $m$, we will prove in the next section that every such graph has a $\left(2, k, m, 64 k m^{2}\right)$ decomposition. This, and Lemma 1, will imply Theorem 1. Indeed, $64 s q=64 k s^{2}$, since we set $s$ to be $q / k$.

In the remainder of this section we prove Lemma 1.

Lemma 1. If $H \in \mathcal{H}(k, n)$ is $(2, k, s, 64 s q)$-decomposable, then $\Gamma(k, n) \supset H$.

Proof of Lemma 1. Let $F_{1}, \ldots, F_{k}$ be a $(2, k, s, 64 s q)$-decomposition of $H$. Define $F_{i}$ for all $i=k+1, \ldots, q$, by setting $F_{t k+j}=F_{j}$ for each $j \in\{1, \ldots, k\}$, and each $t \in\{1, \ldots, s-1\}$. Trivially, for each edge $e \in E(H)$, there are two distinct indices $j^{\prime}, j^{\prime \prime} \in\{1, \ldots, k\}$ such that $e \in F_{t k+j}$ for each $j=j^{\prime}, j^{\prime \prime}$ and $t \in\{0,1, \ldots, s-1\}$.

Let $\mathcal{P}_{i}$ be a family of paths which exhibits the $(s, 64 s q)$-path-separability of $F_{i}, i=1, \ldots, q$. The following fact is crucial.

Claim 2 There exist subsets $E_{i} \subseteq F_{i}, i=1, \ldots, q$, such that

(i) for all $1 \leq i<j \leq q$, we have $E_{i} \cap E_{j}=\emptyset$, and

(ii) for all $i=1, \ldots, q$, we have $E_{i} \cap P \neq \emptyset$, for each $P \in \mathcal{P}_{i}$.

Proof of Claim 2. Consider an auxiliary bipartite graph $B$ with the paths from (the multiset) $\bigcup_{i}^{q} \mathcal{P}_{i}$ on one side (red vertices) and the edges of $H$ on the other (blue vertices), where the edges of $B$ connect the edges of $H$ with the paths they belong to. In this graph, the degree of every red vertex is at least $2 s$ (the 
length of the path), while the degree of every blue vertex is at most $2 s$ (since every edge of $H$ belongs to exactly $2 s$ graphs $F_{i}$ and, for given $i$, to at most one path from $\left.\mathcal{P}_{i}\right)$. Hence, by Hall's matching theorem, one can assign to each path a different edge. The edges assigned to the paths of $\mathcal{P}_{i}$ form the desired set $E_{i}$, $i=1, \ldots, q$.

Continuing with the proof of Lemma 1 , let $E_{i} \subseteq F_{i}, i=1, \ldots, q$, satisfy (i) and (ii) of Claim 2, and let $L_{i}=F_{i} \backslash E_{i}$ for each $i=1, \ldots, q$. Then, clearly,

(a) for each edge $e \in E(H)$, there exist two distinct indices $j^{\prime}, j^{\prime \prime} \in\{1,2, \ldots, k\}$ such that $e \in L_{k t+j}$ for all but at most one of the pairs of integers $(j, t)$, where $j=j^{\prime}, j^{\prime \prime}$ and $t \in\{0, \ldots, s-1\}$, and

(b) each connected component of each $L_{i}$ has at most $64 s q$ vertices.

Recall that $\Gamma(k, n)=\Gamma$ is constructed by blowing up the vertices of another graph $\Gamma^{\prime}(k, n)=\Gamma^{\prime}$. Now, we will show the existence of an embedding $f$ : $V(H) \rightarrow V\left(\Gamma^{\prime}\right)=\{0,1\}^{q}$ such that

(I) if $x y \in H$, then $f(x)=f(y)$, or $f(x) f(y) \in \Gamma^{\prime}$, and

(II) $\left|f^{-1}(v)\right| \leq 64 q^{4} / k$ for each $v \in \Gamma^{\prime}$.

This will prove that $H$ is a subgraph of $\Gamma$.

For each $i=1, \ldots, q$, let $\mathcal{C}_{i}$ denote the set of connected components of $L_{i}$, and let a function $f_{i}: \mathcal{C}_{i} \rightarrow\{0,1\}$ be given. We now specify $f$ : for each $x \in V(H)$, let $f(x)$ be such that the $i$-th coordinate of $f(x)$ is $f_{i}\left(C_{i}(x)\right)$, where $C_{i}(x)$ is the connected component of $L_{i}$ that contains $x$. Observe that if $x y \in L_{i}$, then clearly, $x$ and $y$ are in the same connected component of $L_{i}$, and the $i$-th component of $f(x)$ equals the $i$-th component of $f(y)$. Hence, by (a) and the construction of $\Gamma^{\prime}$, if $x y \in H$ then $f(x) f(y) \in \Gamma^{\prime}$, unless $f(x)=f(y)$. Consequently, $f$ satisfies condition (I).

It remains to show that there exists such an $f$ with $\left|f^{-1}(v)\right| \leq 64 q^{4} / k$ for all $v \in V\left(\Gamma^{\prime}\right)$. We apply the probabilistic method. Let each $f_{i}$ be chosen randomly according to the uniform distribution on $\{0,1\}^{\mathcal{C}_{i}}$. Then $f$ is also random, but not necessarily uniform on $V\left(\Gamma^{\prime}\right)^{V(H)}$. To avoid this problem, we split $V(H)$ suitably, being guided by the following elementary observation.

Claim 3 Let each $f_{i}: X_{i} \rightarrow V$ be drawn uniformly at random, $i=1, \ldots, q$. Let $Y$ be a set of vectors in $X_{1} \times \ldots \times X_{q}$, such that no two vectors in $Y$ have a common coordinate. Then, letting $y_{i}(i=1, \ldots, q)$ denote the $i$-th coordinate of each $y \in X_{1} \times \ldots \times X_{q}$, the function $f: Y \rightarrow V^{q}$, defined by $f(y)=\left(f_{1}\left(y_{1}\right), \ldots, f_{q}\left(y_{q}\right)\right)$ is also drawn according to the uniform distribution on $\left(V^{q}\right)^{Y}$.

Let $H^{\prime}$ be the graph obtained from $H$ by connecting every two vertices $x$ and $y$ which, for some $i=1, \ldots, q$, are in the same connected component of $L_{i}$. If $Y$ is an independent set in $H^{\prime}$, then, by Claim $3,\left.f\right|_{Y}$ is distributed uniformly on $V\left(\Gamma^{\prime}\right)^{Y}$. As the degree of $H^{\prime}$ is smaller than $q(64 q s)=64 s q^{2}$, we can partition the vertices of $H$ into $r=64 s q^{2}=64 q^{3} / k$ sets $Y_{1}, Y_{2}, \ldots, Y_{r}$, each independent in $H^{\prime}$, and so, for each $j=1, \ldots, r,\left.f\right|_{Y_{j}}$ is distributed uniformly on $V\left(\Gamma^{\prime}\right)^{Y_{j}}$.

In fact, by applying the Hajnal-Szemerédi Theorem [14] to $H^{\prime}$, we can ensure that $Y_{1}, \ldots, Y_{r}$ have all equal cardinality (to within 1 ). So each $Y_{j}$ has cardinality 
$n / 64 s q^{2}$. Since $V\left(\Gamma^{\prime}\right)=V$ has cardinality $2^{q}=k n / 8 \log ^{4} n$, which is at least $k n / 10 q^{4}$ for large enough $n$, it follows that $\left|Y_{j}\right| /|V| \leq 5 s k / 32=5 q / 32$.

To confirm condition (II), it suffices to show that, for each fixed $j \in\{1, \ldots, r\}$, with probability at least $1-o(1 / r),\left|f^{-1}(v) \cap Y_{j}\right| \leq q$ for all $v \in V$. Thus, the following simple, probabilistic fact is just what we need.

Claim 4 Let $Y$ and $V$ be two sets with $|Y| \leq 5 q|V| / 32$ and $|V|=2^{q}$. If a function $f: Y \rightarrow V$ is chosen uniformly at random, then

$$
\operatorname{Prob}\left(\exists v \in V:\left|f^{-1}(v)\right|>q\right)=o\left(1 / q^{3}\right) .
$$

Proof of Claim 4. The probability in question can be bounded from above by

$$
|V|\left(\begin{array}{c}
|Y| \\
q
\end{array}\right)|V|^{-q}<|V|\left(\frac{15}{32}\right)^{q}=\left(\frac{15}{16}\right)^{q}=o\left(\frac{1}{q^{3}}\right) .
$$

To finish the proof of Lemma 1 we apply Claim $4 r$ times, with $Y=Y_{j}$, $j=1, \ldots, r$, and $V=V\left(\Gamma^{\prime}\right)$.

\section{Windmill Decomposition of Graphs}

In this section we prove the following proposition which together with Lemma 1 completes the proof of Theorem 1.

Proposition 1. For each $k \geq 3$, every graph $H \in \mathcal{H}(k, n)$ is $(2, k, s, 64 s q)$ decomposable

In view of Examples 2.2-2.4, Proposition 1 is a simple corollary of the next result and the fact that $64 s q=64 s^{2} k$. Recall the definition of a windmill given in Example 2.2. We say that $H \in \mathcal{H}(k, n)$ is $(2, k)$-decomposable into windmills if there exist subgraphs $F_{1}, \ldots, F_{k}$ of $H$, not necessaily all distinct, such that

(i) each edge of $H$ appears in exactly two of the $F_{i}$ 's, and

(ii) each $F_{i}$ is a vertex-disjoint collection of windmills.

In this case, the collection $F_{1}, \ldots, F_{k}$ is called a $(2, k)$-decomposition of $H$ into windmills.

Proposition 2. For each odd $k \geq 3$, every graph $H$ of maximum degree at most $k$ is $(2, k)$-decomposable into windmills.

Proof. We first present a construction of subgraphs $W, F_{2}, \ldots, F_{(k-1) / 2}$ of $H$. Next we prove that each $F_{i}$ is a vertex-disjoint collection of windmills (see Lemma 2, below), and that $W$ is $(2,3)$-decomposable into windmills (see Lemma 2 and Lemma 3).

The construction of $W, F_{2}, \ldots, F_{(k-1) / 2}$. Let us assume, without loss of generality, that $H$ is $k$-regular, as $H$ is a subgraph of a $k$-regular graph (that may have a larger vertex-set). We further assume that $H$ is connected. Recall that a Tutte 
set in $H$ is a set $S$ of vertices such that if $H-S$ has $m$ connected components with an odd number of vertices, then the size of the maximum matching in $H$ is $\frac{1}{2}(|V(H)|-m+|S|)$. Let $S$ be a maximal Tutte set of $H$, and let $\mathcal{C}=\left\{C_{1}, \ldots, C_{m}\right\}$ denote the set of odd connected components of $H \backslash S$. For any $C \in \mathcal{C}$, and any subgraph $F \subseteq H$, let $\delta_{F}(C)$ denote the number of edges in $F$ with exactly one endpoint in $C$.

Using the Gallai-Edmonds Structure Theorem (see, e.g., [15], pp. 94-95) and Hall's Theorem, one can prove that there exists a collection $M^{*}$ of vertex-disjoint stars of $H$ that satisfies the following properties.

(i) Each $s \in S$ is a center of a star $\chi_{s} \in M^{*}$, where the $\chi_{s}$ 's, $s \in S$, are such that

$(\dagger)$ each such $\chi_{s}$ has at least one edge,

(a) no $\chi_{s}$ contains any vertices of $S \backslash\{s\}$,

(b) no $\chi_{s}$ contains any vertices in any even connected component of $H \backslash S$,

(c) for each $C \in \mathcal{C}$, there is exactly one edge $e_{C}$ that is incident to a vertex in $C$, and also belongs to some $\chi_{s}$, and

(d) for each $s \in S$, there is at most one $C \in \mathcal{C}$ such that $\delta_{H}(C) \geq k$ and $V\left(\chi_{s}\right) \cap V(C)$ is nonempty.

(ii) The subgraph of $M^{*}$ induced by the vertices of $H$ not belonging to any $\chi_{s}$ as in (i) is a perfect matching.

Note that

(*) $M^{*}\left[V^{\prime}\right]$ has maximum degree at most 1 for any set $V^{\prime}$ of vertices disjoint from $S$.

Each vertex in $H$ has degree at least 1 in $M^{*}$; let $F_{1}^{\prime}, \ldots, F_{(k-1) / 2}^{\prime}$ be subgraphs with maximum degree at most 2 such that $\bigcup_{j} F_{j}^{\prime}=H \backslash M^{*}$. Such subgraphs exist by the Petersen Theorem (cf. Example 2.4). For each $j \geq 2$ in its turn, we now construct $F_{j}$ from $F_{j}^{\prime}$ as follows. For each $C \in \mathcal{C}$ such that $\delta_{F_{j}^{\prime}}(C)=0$, add $e_{C}$ to $F_{j}^{\prime}$ unless it already belongs to $F_{j^{\prime}}$ for some $2 \leq j^{\prime}<j$, and call the resulting graph $F_{j}$. Note that

$(* *) \delta_{F_{j}}(C) \leq 1$ if $\delta_{F_{j}^{\prime}}(C)=0$, for each $C \in \mathcal{C}$.

Let $W=H \backslash F_{2} \cup \ldots \cup F_{(k-1) / 2}$. (Note that $W \backslash M^{*}=F_{1}^{\prime}$.)

The next two lemmas describe the structure of the subgraphs $W, F_{2}, \ldots$, $F_{(k-1) / 2}$. Their proofs are given in the appendix.

Lemma 2. The graphs $W, F_{2}, \ldots, F_{(k-1) / 2}$ satisfy the following two conditions.

(i) We can partition $V(W)=V(H)$ into sets $V_{0}, \ldots, V_{t}$ such that

(A) for each $j \in\{1, \ldots, t\}$, there is at most one edge $e_{j}$ in $W$ with exactly one endpoint in $V_{j}$ (the edges $e_{j}$ will be called the parting edges of $W$ ), and

(B) each $W\left[V_{i}\right]$ has maximum degree 3, and a matching $M_{i}$ that saturates all vertices of degree 3 in $W\left[V_{i}\right]$.

(ii) Each $F_{i}$ is a vertex-disjoint collection of windmills. 
Lemma 3. Let $H^{\prime}$ be a graph in $\mathcal{H}(3, n)$ that contains a matching $M$ that saturates each vertex of degree 3 in $H^{\prime}$. Then there exist three subgraphs $F_{1}, F_{2}, F_{3}$, such that

(i) each edge of $H^{\prime}$ appears in exactly two of the $F_{i}$ 's;

(ii) $F_{1}, F_{2}$ have maximum degree 2 , and $F_{3}$ is a collection of vertex disjoint windmills.

We now use Lemmas 2 and 3 to finish the proof of Proposition 2. Take two copies of each of the graphs $F_{2}, \ldots, F_{(k-1) / 2}$ to obtain $F_{2}, \ldots, F_{k-2}$, which are each vertex-disjoint collections of windmills, by Lemma 2 . Therefore, to prove Proposition 2 , all we need to show is that $W$ is $(2,3)$-decomposable into windmills, say, $F_{1}, F_{k-1}$, and $F_{k}$, and thus obtain a $(2, k)$-decomposition of $H$ into vertex-disjoint windmills.

To this end we use Lemma 3 . Let $V_{0}, V_{1}, \ldots$ be as in Lemma 2 (i). For each $W\left[V_{j}\right]$, let $Y_{j, 1}, Y_{j_{2}}, Y_{j_{3}}$ be a $(2,3)$-decomposition of $W\left[V_{j}\right]$ into windmills, such that each $Y_{j, 1}$ and $Y_{j, 2}$ have maximum degree 2 ; such graphs exist by Lemma 3 . Let $E^{\prime}$ denote the set of parting edges in $W$. Note that $F_{1}=E^{\prime} \cup\left(\cup_{j} Y_{j, 1}\right)$ and $F_{k-1}=E^{\prime} \cup\left(\bigcup_{j} Y_{j, 2}\right)$ is a vertex-disjoint collection of windmills, and $F_{k}=\bigcup Y_{j, 3}$ is also a vertex-disjoint collection of windmills, and that each edge of $W$ appears in at least 2 of the graphs $F_{1}, F_{k-1}, F_{k}$. This completes the proof of Proposition 2.

\section{Universal Graphs with Fewer Vertices}

In this section, we sketch a construction of an $\mathcal{H}(k, n)$-universal graph $\Lambda(k, n)=$ $\Lambda$, which still has $O\left(n^{2-\frac{2}{k}}(\log n)^{1+8 / k}\right)$ edges, but only has $(1+\epsilon) n$ vertices, for any fixed $\epsilon>0$.

Let us write $V(\Gamma(k, n))=V$, and let $\Omega=(V, Q, E)$ be a bipartite graph of bounded degree such that $|Q|=(1+\epsilon) n$, and $|N(X)| \geq|X|$ for each subset $X \subset V$ such that $|X| \leq n$. It is well-known that such an $\Omega$, usually called a concentrator, exists, and can be constructed explicitly using the known constructions of bounded-degree expanders. We now construct $\Lambda(k, n)$, which has $Q$ as its vertex-set. Let $\nu$ and $\nu^{\prime}$ be vertices in $Q$. The edge $\nu \nu^{\prime} \in \Lambda$ if and only if there exist vertices $v, v^{\prime} \in V$ such that $v v^{\prime} \in \Gamma(k, n)$, and $v \nu, v^{\prime} \nu^{\prime} \in \Omega$. We have $|E(\Lambda)| \leq|E(\Gamma)| \Delta(\Omega)^{2}=O(|E(\Gamma)|)$.

The following theorem can be easily deduced from Theorem 1.1.

Theorem 5. $\Lambda(k, n)$ is $\mathcal{H}(k, n)$-universal for all $k \geq 3$, and $n$ sufficiently large.

Proof. Let $H \in \mathcal{H}(k, n)$. Then, by Theorem 1.1, $H \subset \Gamma(k, n)$. By the expanding property of $\Omega$ and by Hall's Theorem, $\Omega$ has a matching $f$ between $V(H)$ and a subset of $Q$. Thus, if $x y \in H$ then $f(x) f(y) \in \Lambda$.

\section{A More Details for Section 3}

Proof of Lemma 2: We first prove (ii), namely, show that each $F_{i}$ is indeed a vertex-disjoint collection of windmills. As $F_{i}^{\prime}$ is a collection of vertex-disjoint 
cycles and paths, each connected component of $F_{i}$ not containing an edge of $F_{i} \backslash F_{i}^{\prime}$ is either a path or a cycle. Thus, it remains to show that $L$ is a windmill, for each $L$ that is a connected component of $F_{i}$ containing an edge $e=x y$ in $F_{i} \backslash F_{i}^{\prime}$. The edge $e$ is of the form $e_{C}$, for some $C \in \mathcal{C}$; let us assume without loss of generality that $y \notin C$, but $x \in C$. Note that (a') both $x$ and $y$ cannot be in the same connected component of $F_{i}^{\prime}$, since otherwise $\delta_{F^{\prime}}(C)>0$, and $e \notin F_{i}$. Similarly, (b') the connected component $L_{x}^{\prime}$ of $F_{i}^{\prime}$ containing $x$ must be contained in $C$. But (a'), (b') and $\left({ }^{* *}\right)$, together with the fact that each connected component of $F_{i}^{\prime}$ is either a path or cycle, imply that $L$ must be a windmill. Thus Lemma 2 (ii) follows.

We now show that $W$ satisfies (i) of Lemma 2 .

Claim 3.1: For each $C \in \mathcal{C}$, the quantity $\delta_{H}(C)$ is an odd integer. So if $\delta_{H}(C)<k$, then $\delta_{H}(C) \leq k-2$.

Claim 3.1 follows from the fact that $H$ is $k$-regular, with $k$ an odd integer, and that $C$ has an odd number of vertices.

Claim 3.2: For each $C \in \mathcal{C}$, and each $i$, the quantity $\delta_{F_{i}^{\prime}}(C)$ is an even integer. So if $\delta_{F_{i}^{\prime}}(C)>0$, then $\delta_{F_{i}^{\prime}}(C) \geq 2$.

Claim 3.2 follows from the fact that each vertex in $C$ has degree exactly 1 in $M^{*}$, so each vertex in $C$ has degree exactly $k-1$ in $H \backslash M^{*}$, and so exactly 2 in each $F_{i}^{\prime}$.

Claim 3.3: All but one of $s$ 's neighbors in $M^{*} \cap W$ are in some $C \in \mathcal{C}$ such that $\delta_{W}(C) \leq 1$.

Proof of Claim 3.3: From the definition of $M^{*}$ and Claim 3.1, all but one of $s$ 's neighbors in $M^{*}$ are in some $C \in \mathcal{C}$ such that $\delta_{H}(C) \leq k-2$. But by definition of the $F_{i}$ 's, and Claim 3.2, for each such $C$, either $\delta_{H \backslash\left(F_{2} \cup \ldots \cup F_{i}\right)}(C) \leq$ $\delta_{H \backslash\left(F_{2} \cup \ldots \cup F_{i-1}\right)}(C)-2$ for each $i$, or $e_{C} \in F_{i}$, and therefore, $e_{C} \notin W$, and so Claim 3.3 follows.

Claim 3.4: Let $V_{0}$ be the set of vertices $v$ such that either $v \in S$, or $v \in C \in \mathcal{C}$ such that $\delta_{W}(C)>1$, or $v$ is in any even-sized connected component of $H \backslash S$. Then (1) each vertex in $W\left[V_{0}\right]$ has degree at most 3, and (2) $W\left[V_{0}\right]$ has a matching covering all vertices of degree 3 in $W\left[V_{0}\right]$.

Proof of Claim 3.4: By Claim 3.3, $M^{*}\left[V_{0}\right]$ is a matching. Since $W\left[V_{0}\right] \backslash M^{*} \subseteq F_{1}^{\prime}$, which has maximum degree 2, both (1) and (2) follow.

Claim 3.5: For each $C_{i_{j}} \in \mathcal{C}^{\prime}=\left\{C_{i_{1}}, \ldots, C_{i_{l}}\right\} \subseteq \mathcal{C}$ such that $\delta_{W}\left(C_{i_{j}}\right) \leq 1$ for each $j \in\{1, . ., l\}$, let $V_{j}$ denote the set of vertices of $C_{i_{j}}$. The graph $W\left[V_{j}\right]$ has a matching that saturates all vertices of degree 3 in $W\left[V_{j}\right]$.

Proof of Claim 3.5: $M^{*}\left[V_{j}\right]$ is a matching by $\left(^{*}\right)$, and $W\left[V_{0}\right] \backslash M^{*} \subseteq F_{1}^{\prime}$, which has maximum degree 2 .

Lemma 2 follows from Claim 3.6.

Claim 3.6: $W$ satisfies (i) of Lemma 2.

Proof of Claim 3.6: Use Claims 3.4 and 3.5.

Proof of Lemma 3: The idea is to find a subset $M_{1}$ of $M$, and a matching $M_{2}$ that is a subset of $H^{\prime} \backslash M$, such that $F_{2}=\left(M \backslash M_{1}\right) \cup\left(\left(H^{\prime} \backslash M\right) \backslash M_{2}\right)$ has maximum 
degree 2, and $F_{3}=\left(H^{\prime} \backslash M\right) \cup M_{1}$ is a vertex-disjoint collection of windmills. Then let

$$
F_{1}=M \cup M_{2} ; F_{2}=\left(M \backslash M_{1}\right) \cup\left(\left(H^{\prime} \backslash M\right) \backslash M_{2}\right) ; F_{3}=\left(H^{\prime} \backslash M\right) \cup M_{1} .
$$

Each edge of $H^{\prime}$ appears in exactly two of the $F_{i}$ 's, and hence this will imply Lemma 3.

We now describe how to find $M_{1}$ and $M_{2}$. Let us add edges to $H^{\prime} \backslash M$ to obtain a graph $F_{3}$, consisting of vertex-disjoint windmills, such that

(i) the units of the windmills in $F_{3}$ are the connected components of $H^{\prime} \backslash M$, and

(ii) each odd cycle $C$ of $H^{\prime} \backslash M$ such that each $v \in C$ has degree 3, is a unit of a windmill in $F_{3}$ with at least two units.

To find such an $F_{3}$, contract each connected component $C$ of $H^{\prime} \backslash M$ to a single vertex $v_{C}$; call the resulting graph $G$, and take any subgraph of $G$ with the fewest possible edges such that each such $v_{C}$ has positive degree if $v_{C}$ has positive degree in $G$; the resulting graph corresponds to such an $F_{3}$. Let the set of edges that are the spokes of the windmills in $F_{3}$ be $M_{1}$; note that $M_{1} \subseteq M$, and that $F_{3}=\left(H^{\prime} \backslash M\right) \cup M_{1}$. Note also that each odd cycle of $H^{\prime} \backslash M$ contains a vertex $v$ such that $v$ has degree exactly 2 in $H^{\prime} \backslash M_{1}$, and degree exactly zero in $M \backslash M_{1}$.

Let us now specify $M_{2} \subseteq H^{\prime} \backslash M$. Let $C$ be a connected component of $H^{\prime} \backslash M$. If $C$ is a path or an even cycle, let $M_{2} \cap C$ be any matching such that $C \backslash M_{2}$ is also a matching. If $C$ is an odd cycle, let $v$ be a vertex in $C$ that has degree exactly 2 in $H^{\prime} \backslash M_{1}$, and let $M_{2}$ be a matching of $C$ such that the only vertex of $C$ that has degree 2 in $C \backslash M_{2}$ is $v$.

One can check that the maximum degree of each vertex in $F_{1}$ and in $F_{2}$ is 2 ; Indeed, $F_{1}$ is the union of two matchings. Each vertex on a path or even cycle $C$ of $H^{\prime} \backslash M$ has degree at most 1 in $M \backslash M_{1}$, and degree at most 1 in $\left(H^{\prime} \backslash M\right) \backslash M_{2}$, while each vertex on an odd cycle $C$ of $H^{\prime} \backslash M$ having degree 2 in $\left(H^{\prime} \backslash M\right) \backslash M_{2}$ has degree 0 in $M \backslash M_{1}$. Because each vertex of $H^{\prime}$ has degree at most 2 in $H^{\prime} \backslash M$, each vertex of $H^{\prime}$ has degree at most 2 in $F_{2}$. Finally, as we have already established that $F_{3}$ is a collection of vertex-disjoint windmills, Lemma 3 follows.

\section{References}

1. N. Alon and V. Asodi, Sparse universal graphs, Journal of Computational and Applied Mathematics, to appear.

2. N. Alon, M. Capalbo, Y. Kohayakawa, V. Rödl, A. Ruciński, and E. Szemerédi, Universality and tolerance, Proceedings of the 41st IEEE Annual Symposium on FOCS, pp. 14-21, 2000.

3. L. Babai, F. R. K. Chung, P. Erdős, R. L. Graham, J. Spencer, On graphs which contain all sparse graphs, Ann. Discrete Math., 12 (1982), pp. 21-26.

4. S. N. Bhatt, F. Chung, F. T. Leighton and A. Rosenberg, Universal graphs for bounded-degree trees and planar graphs, SIAM J. Disc. Math. 2 (1989), 145-155. 
5. S. N. Bhatt and C. E. Leiserson, How to assemble tree machines, Advances in Computing Research, F. Preparata, ed., 1984.

6. M. Capalbo, A small universal graph for bounded-degree planar graphs, SODA (1999), 150-154.

7. F. R. K. Chung and R. L. Graham, On graphs which contain all small trees, J. Combin. Theory Ser. B, 24 (1978) pp. 14-23.

8. F. R. K. Chung and R. L. Graham, On universal graphs, Ann. New York Acad. Sci., 319 (1979) pp. 136-140.

9. F. R. K. Chung and R. L. Graham, On universal graphs for spanning trees, Proc. London Math. Soc., 27 (1983) pp. 203-211.

10. F. R. K. Chung, R. L. Graham, and N. Pippenger, On graphs which contain all small trees II, Proc. 1976 Hungarian Colloquium on Combinatorics, 1978, pp. 213223.

11. M. Capalbo and S. R. Kosaraju, Small universal graphs, STOC (1999), 741-749.

12. F. R. K. Chung, A. L. Rosenberg, and L. Snyder, Perfect storage representations for families of data structures, SIAM J. Alg. Disc. Methods., 4 (1983), pp. 548-565.

13. J. Friedman and N. Pippenger, Expanding graphs contain all small trees, Combinatorica, 7 (1987), pp. 71-76.

14. A. Hajnal and E. Szemerédi, Proof of a conjecture of Erdős, in Combinatorial Theory and its Applications, Vol. II (P. Erdős, A. Rényi, and V. T. Sós, eds.), Colloq. Math Soc. J. Bolyai 4, North Holland, Amsterdam 1970, 601-623.

15. L. Lovász and M. D. Plummer, Matching Theory, North Holland, Amsterdam (1986).

16. V. Rödl, A note on universal graphs, Ars Combin., 11 (1981), 225-229. 